Original Research Paper

\title{
Topological Beth Model and its Application to Functionals of High Types
}

\author{
Farida Kachapova \\ Department of Mathematical Sciences, Auckland University of Technology, Auckland, New Zealand
}

Article history

Received: 6-06-2020

Revised: 29-07-2020

Accepted: 13-10-2020

Email: farida.kachapova@aut.ac.nz

\begin{abstract}
Based on the definition of Beth-Kripke model by Dragalin, we describe Beth model from the topological point of view. We show the relation of the topological definition with more traditional relational definition of Beth model that is based on forcing. We apply the topological definition to construct a Beth model for a theory of intuitionistic functionals of high types and to prove its consistency.
\end{abstract}

Keywords: Intuitionistic Functional, Creating Subject, Topological Model, Beth Model, Forcing Relation.

\section{Introduction}

The studies of metamathematical properties of nonclassical theories are based on a variety of models such as topological models, Beth model and Kripke model. Moschovakis developed a topological model (Moschovakis, 1973) for intuitionistic second-order arithmetic and developed semantics (Moschovakis, $1987)$ for a theory of lawless sequences. Van Dalen (1978) constructed a Beth model for intuitionistic analysis. In his book (Dragalin, 1987) Dragalin studied a general Beth-Kripke model ( $B K$-model) that combines forcing from Beth model and realizability from Kripke model. The applications of $B K$-model in (Dragalin, 1987) include different versions of intuitionistic arithmetic and analysis. Most applications of the aforementioned models are for intuitionistic sequences of natural numbers (e.g. choice sequences and lawless sequences). In (Kachapova, 2014; 2015) we created a Beth model for intuitionistic functionals of high types: 1functionals (sequences of natural numbers), 2functionals (sequences of 1 -functionals), ..., $(n+1)$ functionals (sequences of $n$-functionals). That model was based on the relational definition of Beth model by van Dalen (1978).

In this study we describe the general concept of Beth model from the topological point of view. The topological version has a simpler definition than the relational version and is consistent with the definition of a general algebraic model for an axiomatic theory as in (Dragalin, 1987). In this study we apply the topological version of Beth model to the intuitionistic theory SLP of high-order functionals from (Kachapova, 2015), including lawless functionals and the "creating subject". It can be seen that the topological version of Beth model simplifies some constructions and consistency proofs. In metamathematical proofs we use classical logic.

\section{Preliminary Concepts}

The introductory theory in this section follows the book by Dragalin (1987).

\subsection{Logical-Mathematical Language. Axiomatic Theory}

Definition 2.1

A logical-mathematical language of first order (or logical-mathematical language, or just language in short) is defined as a sequence $\Omega=\langle$ Srt, Cnst, Fn, Pr $\rangle$, where

1) Srt is a non-empty set of sorts of objects and for each sort $\pi \in S r t$ there is a countable collection of variables of this sort;

2) Cnst is the set of all constants of the language;

3) $F n$ is the set of all functional symbols of the language;

4) $P r$ is the set of all predicate symbols of the language.

In the language $\Omega$ we can construct terms, atomic formulas and formulas as usual.

\section{Definition 2.2}

Axiomatic theory (or just theory in short) is defined as $T h=\langle\Omega, l, A\rangle$, where each of the three objects is described as follows.

1) $\Omega$ is a logical-mathematical language.

2) $l$ is the logic of the theory. We will use only the intuitionistic logic $H P C$ (Heyting's predicate calculus). 
3) $A$ is some set of closed formulas (i.e., formulas without parameters) of the language $\Omega$; it is called the set of non-logical axioms of $T h$. When axioms are stated as non-closed formulas, it means that they must be closed by universal quantifiers over all parameters.

The notation $T h \vdash \varphi$ (formula $\varphi$ is derivable in the theory $T h$ ) means that $\varphi$ is derivable in the logic $l$ from a finite subset of the axiom set $A$.

\subsection{Pseudo Boolean Algebras}

\section{Definition 2.3}

A pair $\langle B, \leq\rangle$ is called a pseudo Boolean algebra (p.B.a.) if $B$ is a set, $\leq$ is a binary relation on $B$ and they satisfy the following 9 conditions.

1) $a \leq a$.

2) $a \leq b \& b \leq c \Rightarrow a \leq c$.

For any $a, b \in B$ there exists an element $a \wedge b \in B$ such that:

3) $a \wedge b \leq a, a \wedge b \leq b$,

4) $c \leq a \& c \leq b \Rightarrow c \leq a \wedge b$.

For any $a, b \in B$ there exists an element $a \vee b \in B$ such that:

5) $a \leq a \vee b, b \leq a \vee b$,

6) $a \leq c \& b \leq c \Rightarrow a \vee b \leq c$.

For any $a, b \in B$ there exists an element $(a \supset b) \in B$ such that:

7) $a \wedge(a \supset b) \leq b$,

8) $c \wedge a \leq b \Rightarrow c \leq(a \supset b)$.

9) There exists an element $\perp \in B$ such that for any $a \in$ $B: \perp \leq a$.

The element $\perp$ is "falsity" and the element $T=(\perp \supset$ $\perp$ ) is "truth".

\section{Definition 2.4}

Suppose $\langle B, \leq\rangle$ is a p.B.a., $W \subseteq B$ and $a \in B$.

1) $a$ is denoted $\wedge W$ and is called the intersection or conjunction of $W$ if
a) $(\forall c \in W)(a \leq c)$ and
b) for any $d \in B,(\forall c \in W)(d \leq c) \Rightarrow d \leq a$.

2) $a$ is denoted $\vee W$ and is called the union or disjunction of $W$ if

a) $(\forall c \in W)(c \leq a)$ and

b) for any $d \in B,(\forall c \in W)(c \leq d) \Rightarrow a \leq d$.

\section{Definition 2.5}

A p.B.a. $\langle B, \leq\rangle$ is called complete if for any $W \subseteq B$ there exist $\wedge W \in B$ and $\vee W \in B$.

\subsection{Algebraic Model of a Language}

The following is definition of an algebraic model with constant domains. For brevity we call it just an algebraic model.

\section{Definition 2.6}

An algebraic model of the language $\Omega$ is a sequence $A=\langle B, D, \widehat{\text { Cnst }}, \widehat{F n}, \widehat{P r}\rangle$ of objects defined as follows.

1) $B$ is a complete p.B.a.

2) To each sort $\pi$ of the language $\Omega$ the function $D$ assigns a non-empty set $D_{\pi}$, which is called the domain of elements of sort $\pi$.

3) To each constant of sort $\pi$ the function $\widehat{\text { Cnst }}$ assigns an element $\bar{c} \in D_{\pi}$.

4) Function $\widehat{F n}$ assigns values to functional symbols of $\Omega$ in the following way.

Let $f$ be a functional symbol of sort $\pi$ with arguments of sorts $\pi_{1}, \ldots, \pi_{k}$. Then for any $q_{1} \in D_{\pi_{1}}, \ldots, q_{k} \in D_{\pi_{k}}$, $q \in D_{\pi}, \widehat{F n}\left(f, q_{1}, \ldots, q_{k}, q\right) \in B$.

$\widehat{F n}$ satisfies the following conditions (1) and (2).

$\vee\left\{\widehat{F n}\left(f, q_{1}, \ldots, q_{k}, q\right) \mid q \in D_{\pi}\right\}=T$.

If $q \neq q^{\prime}$, then:

$\widehat{F n}\left(f, q_{1}, \ldots, q_{k}, q\right) \wedge \widehat{F n}\left(f, q_{1}, \ldots, q_{k}, q^{\prime}\right)=\perp$.

5) If $P$ is a predicate symbol of $\Omega$ with arguments of sorts $\pi_{1}, \ldots, \pi_{k}$, then for any $q_{1} \in D_{\pi_{1}}, \ldots, q_{k} \in D_{\pi_{k}}$ :

$\widehat{\operatorname{Pr}}\left(P, q_{1}, \ldots, q_{k}\right) \in B$.

An evaluated term is a term of the language $\Omega$, in which all parameters are replaced by elements from suitable domains. An evaluated formula is a formula of the language $\Omega$, in which all parameters are replaced by elements from suitable domains.

\section{Definition 2.7}

Suppose $t$ is an evaluated term of sort $\pi$ and $q \in D_{\pi}$. The set $\|t \sim q\|$ is defined by induction on the complexity of $t$. 
1) If $t$ is an element of $D_{\pi}$, then

$$
\|t \sim q\|=\left\{\begin{array}{c}
T \text { if } t=q, \\
\perp \text { otherwise }
\end{array}\right.
$$

2) If $t$ is a constant $c$ and $\bar{c}=\widehat{\operatorname{Cnst}}(c)$, then

$$
\|t \sim q\|=\left\{\begin{array}{c}
T \text { if } \bar{c}=q \\
\perp \text { otherwise }
\end{array}\right.
$$

3) Suppose $t$ is $f\left(t_{1}, \ldots, t_{k}\right)$, where each $t_{i}$ is an evaluated term of sort $\pi_{i}, i=1, \ldots, k$. Then

$$
\begin{gathered}
\|t \sim q\|=\vee\left\{\widehat{F n}\left(f, q_{1}, \ldots, q_{k}, q\right) \wedge\left\|t_{1} \sim q_{1}\right\| \wedge \ldots\right. \\
\left.\wedge\left\|t_{k} \sim q_{k}\right\| \mid q_{1} \in D_{\pi_{1}}, \ldots, q_{k} \in D_{\pi_{k}}\right\} .
\end{gathered}
$$

\section{Lemma 2.8}

For an evaluated term $t$ of sort $\pi$ :

$$
\bigvee\left\{\|t \sim q\| \mid q \in D_{\pi}\right\}=T
$$

\section{Definition 2.9}

For an evaluated formula $\varphi,\|\varphi\|$ is defined by induction on the complexity of $\varphi$.

1) Suppose $\varphi$ is an atomic formula $P\left(t_{1}, \ldots, t_{k}\right)$, where each $t_{i}$ is an evaluated term of sort $\pi_{i}, i=1, \ldots, k$. Then

$$
\begin{aligned}
\|\varphi\|=\vee\left\{\widehat{\operatorname{Pr}}\left(P, q_{1}, \ldots, q_{k}\right) \wedge\left\|t_{1} \sim q_{1}\right\| \wedge \ldots\right. \\
\left.\wedge\left\|t_{k} \sim q_{k}\right\| \mid q_{1} \in D_{\pi_{1}}, \ldots, q_{k} \in D_{\pi_{k}}\right\} .
\end{aligned}
$$

In particular, if each $t_{i}$ is an element $p_{i} \in D_{\pi_{i}}$, then $\|\varphi\|=\widehat{P r}\left(P, p_{1}, \ldots, p_{k}\right)$.

2) $\|\psi \wedge \eta\|=\|\psi\| \wedge\|\eta\|$.

3) $\|\psi \vee \eta\|=\|\psi\| \vee\|\eta\|$.

4) $\|\psi \supset \eta\|=\|\psi\| \supset\|\eta\|$.

5) $\|\perp\|=\perp$.

6) $\|\forall x \psi(x)\|=\wedge\left\{\|\psi(q)\| \mid q \in D_{\pi}\right\}$, where $x$ is a variable of sort $\pi$.

7) $\|\exists x \psi(x)\|=\vee\left\{\|\psi(q)\| \mid q \in D_{\pi}\right\}$, where $x$ is a variable of sort $\pi$.

\section{Theorem 2.10. Soundness Theorem.}

Suppose $\varphi$ is a formula of $\Omega$ and $\varphi^{\prime}$ is $\varphi$ evaluated by elements of appropriate domains. Then the following hold.
1) $H P C \vdash \varphi \Rightarrow\left\|\varphi^{\prime}\right\|=T$.

2) If $\varphi$ is a closed formula, then

$$
H P C \vdash \varphi \Rightarrow\|\varphi\|=T .
$$

\subsection{Topological Space}

\section{Definition 2.11}

A pair $Y=\langle X, \mathrm{~S}\rangle$ is called a topological space if it satisfies the following conditions:

1) $S$ is a collection of subsets of $X$,

2) $\varnothing \in S, X \in S$,

3) $A, B \in S \Rightarrow A \cap B \in S$,

4) $W \subseteq S \Rightarrow \cup W \in S$.

Elements of $S$ are called open sets and $S$ is called the topology on $X$.

\section{Definition 2.12}

Suppose $Y=\langle X, S\rangle$ is a topological space. A collection $H$ of subsets of $X$ is called a base of the topology $S$ if every $A$ $\in S$ can be written as a union of elements of $H$.

Then we say that $H$ generates the topology $S$.

\section{Lemma 2.13}

Suppose $H$ is a collection of subsets of $X . H$ is a base of some topology on $X$ if it satisfies the following two conditions:

1) $X=\bigcup H$,

2) $\left(\forall A_{1}, A_{2} \in H\right)\left(\forall x \in A_{1} \cap A_{2}\right)\left(\exists A_{3} \in H\right)\left(x \in A_{3} \subseteq A_{1} \cap A_{2}\right)$.

\section{Lemma 2.14}

Suppose $Y=\langle X, S\rangle$ is a topological space. Then $\langle S, \subseteq\rangle$ is a complete p.B.a. with the operations given by:

$$
\begin{aligned}
& \perp=\varnothing, \quad T=X, \\
& A \wedge B=A \cap B, \quad A \vee B=A \cup B, \\
& A \supset B=\{x \in X \mid(\exists C \in S)(x \in C \& C \cap A \subseteq B)\}, \\
& \wedge W=\cap W, \vee W=\bigcup W .
\end{aligned}
$$

\subsection{Completion Operator}

\section{Definition 2.15}

Suppose $Y=\langle B, \leq\rangle$ is a p.B.a. and $\mathfrak{C}: B \rightarrow B$.

1) $\mathfrak{C}$ is called a completion operator on $Y$ if the following 4 conditions are satisfied for any $a, b \in B$ : 

a) $a \leq \mathfrak{C} a$,
b) $a \leq b \Rightarrow \mathfrak{C} a \leq \mathfrak{C} b$,
c) $\mathfrak{C} \mathfrak{C} a=\mathfrak{C} a$,
d) $a=\mathfrak{C} a \wedge a \leq \mathfrak{C} b \Rightarrow a \leq \mathfrak{C}(a \wedge b)$.

2) An element $a \in B$ is called complete if $\mathfrak{C} a=a$.

3) Denote $C l(B)$ the set of all complete elements of $B$.

\section{Lemma 2.16}

Suppose $Y=\langle B, \leq\rangle$ is a p.B.a. and $\mathfrak{C}$ is a completion operator on $Y$. Denote $Y^{+}=\langle C l(B), \leq\rangle$. Then $Y^{+}$is also a p.B.a. with the operations given by:

$$
\begin{aligned}
& \perp^{+}=\mathfrak{C}(\perp), \\
& a \wedge^{+} b=a \wedge b, \\
& a \vee^{+} b=\mathfrak{C}(a \vee b), \\
& a \supset^{+} b=a \supset b .
\end{aligned}
$$

If $Y$ is a complete p.B.a., then $Y^{+}$is also a complete p.B.a. and

$$
\begin{gathered}
\wedge^{+} W=\wedge W, \\
\vee^{+} W=\mathfrak{c}(\vee W) .
\end{gathered}
$$

\section{Beth Model}

The following definitions of Beth frame, Beth algebra and Beth model are modified from the definitions of Beth-Kripke frame, Beth-Kripke algebra and BethKripke model given in the book (Dragalin, 1987). Some accompanying lemmas and theorems are proven here; other proofs can be found in (Dragalin, 1987).

\subsection{Beth Frame}

A tree is a set $M$ with partial order $\leq$ such that for any $x \in M$ the set $\{y \in M \mid y>x\}$ is a well-ordered set. We fix a tree $\langle M, \leq\rangle$ till the end of this section.

\section{Definition 3.1}

1) A path in $M$ is a maximal linearly ordered subset of $M$.

2) A path through an element $x$ is a path containing $x$.

3) For any $a \in M$ denote

$$
\boldsymbol{Q}(a)=\{\text { all paths through } a\} .
$$

The triple $\langle M, \leq, \boldsymbol{Q}\rangle$ is called a Beth frame.

\subsection{Beth Algebra}

\section{Definition 3.2}

1) For any $x \in M,[x]=\{y \in M \mid y \leq x\}$ is called the peaked cone generated by $x$
2) Collection of all sets $[x], x \in M$, satisfies the conditions of Lemma 2.13, therefore it is a base of a topology on $M$. This topology is called the order topology

3) Thus, we get a topological space $\langle M, O p(M)\rangle$, where $O p(M)$ denotes the set of all open subsets of $M$ in the order topology

By Lemma 2.14, $\langle O p(M), \subseteq\rangle$ is a complete p.B.a.

\section{Lemma 3.3}

For any subset $A$ of $M$ :

$$
\begin{gathered}
A \text { is open in the order topology } \\
\Leftrightarrow(\forall a \in A)(\forall b \in M)(b \leq a \Rightarrow b \in A) .
\end{gathered}
$$

Proof

Follows from the definition. $\square$

Definition 3.4

For any $U \subseteq M$ denote:

$$
\boldsymbol{C} U=\{a \in M \mid(\forall S \in \boldsymbol{Q}(a))(S \cap U \neq \varnothing)\} .
$$

\section{Lemma 3.5}

The operator $\boldsymbol{C}$ defined above has the following properties.

1) $C: O p(M) \rightarrow O p(M)$.

2) $C$ is a completion operator on the p.B.a. $\langle O p(M), \subseteq\rangle$.

3) For any open subsets $A$ and $B$ of the set $M$ :

$$
\boldsymbol{C} A \cap \boldsymbol{C} B=\boldsymbol{C}(A \cap B) .
$$

\section{Definition 3.6}

Denote $Y=\langle O p(M), \subseteq\rangle$. It is a complete p.B.a. The operator $\boldsymbol{C}$ from Definition 3.4 is a completion operator.

Thus, by Lemma $2.16, Y^{+}=\langle C l(O p(M)), \subseteq\rangle$ is a complete p.B.a. It is denoted $\boldsymbol{B}(M, \leq, \boldsymbol{Q})$ and is called the Beth algebra generated by the Beth frame $\langle M, \leq, \boldsymbol{Q}\rangle$.

\section{Theorem 3.7. Operations in Beth Algebra.}

Operations in the Beth algebra are given by:

1) $U \wedge V=U \cap V$,

2) $U \vee V=C(U \cup V)$,

3) $U \supset V=\{a \in M \mid(\forall b \leq a)(b \in U \Rightarrow b \in V)\}$,

4) $T=M$,

5) $\perp=\{a \in M \mid Q(a)=\varnothing\}$,

6) $\wedge W=\cap W$

7) $\vee W=\boldsymbol{C}(\mathrm{U} W)$ 


\section{Proof}

Follows from Lemma 2.16. $\square$

\section{Lemma 3.8}

1) For any subsets $A$ and $B$ of the set $M$ :

$$
\boldsymbol{C} A \vee \boldsymbol{C} B=\boldsymbol{C}(A \cup B)
$$

2) For any collection $W$ of subsets of $M$ :

$$
\vee\{\boldsymbol{C} A \mid A \in W\}=\boldsymbol{C}(\bigcup W)
$$

\section{Proof}

1) We need to prove:

$$
\boldsymbol{C}(\boldsymbol{C} A \cup \boldsymbol{C B})=\boldsymbol{C}(A \cup B) .
$$

By properties of completion operator, $A \subseteq C A, A \cup B$ $\subseteq \boldsymbol{C} A \cup \boldsymbol{C} B$ and $\boldsymbol{C}(A \cup B) \subseteq \boldsymbol{C}(\boldsymbol{C} A \cup \boldsymbol{C} B)$.

To prove the inverse, it is sufficient to show that $C A \cup C B \subseteq C(A \cup B)$.

Since $A \subseteq A \cup B$, we have $C A \subseteq C(A \cup B)$. Similarly $\boldsymbol{C} B \subseteq \boldsymbol{C}(A \cup B)$ and $\boldsymbol{C} A \cup \boldsymbol{C} B \subseteq \boldsymbol{C}(A \cup B)$.

2) is proven similarly.

\subsection{Beth Model}

Beth model is a particular case of an algebraic model.

We will use the notation $f: a \rightleftharpoons b$, which means that $f$ is a partial function from set $a$ to set $b$. The notation $Z \downarrow$ means that the object $Z$ is defined.

\section{Definition 3.9}

A Beth model for a language $\Omega=\langle$ Srt, Cnst, Fn, Pr $\rangle$ is an algebraic model $\langle B, D, \widehat{C n s t}, \widehat{F n}, \widehat{P r}\rangle$ with specific definitions of $B, \widehat{F n}$ and $\widehat{P r}$.

1) $B=\boldsymbol{B}(M, \leq, \boldsymbol{Q})$ is the Beth algebra generated by the Beth frame $\langle M, \leq, \boldsymbol{Q}\rangle$.

Elements of $M$ are denoted $\alpha, \beta, \gamma, \ldots$.

2) Before defining $\widehat{F n}$ we define a function $\overline{F n}$.

To each $\alpha \in M$ and each functional symbol $f\left(x_{1}, \ldots, x_{k}\right)$ with sort $\pi$ and arguments of sorts $\pi_{1}, \ldots$, $\pi_{k}$, respectively, the function $\overline{F n}$ assigns a partial function $f^{[\alpha]}: D_{\pi_{1}} \times \ldots \times D_{\pi_{k}} \rightleftharpoons D_{\pi}$ such that for any $q_{1} \in D_{\pi_{1}}, \ldots, q_{k} \in D_{\pi_{k}}, q \in D_{\pi}$ : $\left\{\alpha \mid f^{[\alpha]}\left(q_{1}, \ldots, q_{k}\right)=q\right\}$ is an open set and

$\boldsymbol{C}\left(\left\{\alpha \mid f^{[\alpha]}\left(q_{1}, \ldots q_{k}\right) \downarrow\right\}\right)=M$,

where $C$ is from Definition 3.4.

$\widehat{F n}$ is defined by:

$$
\widehat{F n}\left(f, q_{1}, \ldots, q_{k}, q\right)=\boldsymbol{C}\left(\left\{\alpha \mid f^{[\alpha]}\left(q_{1}, \ldots, q_{k}\right)=q\right\}\right) .
$$

Clearly, $\widehat{F n}$ satisfies the conditions (1) and (2) of the Definition 2.6 of an algebraic model.

3) Before defining $\widehat{P r}$ we define a function $\overline{P r}$.

To each predicate symbol $P\left(x_{1}, \ldots, x_{k}\right)$ with arguments of sorts $\pi_{1}, \ldots, \pi_{k}$, respectively and $q_{1} \in D_{\pi_{1}}, \ldots, q_{k} \in D_{\pi_{k}}$ the function $\overline{P r}$ assigns an open set $\overline{P r}\left(P, q_{1}, \ldots, q_{k}\right)$.

$\widehat{P r}$ is defined by:

$$
\widehat{\operatorname{Pr}}\left(P, q_{1}, \ldots, q_{k}\right)=\boldsymbol{C}\left(\overline{\operatorname{Pr}}\left(P, q_{1}, \ldots, q_{k}\right)\right) .
$$

Clearly, $\widehat{P r}$ satisfies the condition (3) of the Definition 2.6 of an algebraic model.

This completes the definition of Beth model.

\section{Notes}

1) The component $B$ of the Beth model is uniquely defined by the tree $\langle M, \leq\rangle$.

2) The component $\widehat{F n}$ is uniquely defined by the function $\overline{F n}$.

3) The component $\widehat{P r}$ is uniquely defined by the function $\overline{P r}$.

4) Thus, to construct a Beth model, one needs to specify a tree $\langle M, \leq\rangle$, a domain function $D$ and functions $\widehat{C n s t}, \overline{F n}$ and $\overline{P r}$.

\section{Definition 3.10}

For any $\alpha$ and evaluated term $t$ of sort $\pi$ we define $t^{[\alpha]}$ by induction on the complexity of $t$.

1) If $t$ is an element of $D_{\pi}$, then $t^{[\alpha]}=t$.

2) If $t$ is a constant $c$, then $t^{[\alpha]}=\widehat{\operatorname{Cnst}}(c)=\bar{c}$.

3) If $t$ is $f\left(t_{1}, \ldots, t_{k}\right)$, then $t^{[\alpha]}=f^{[\alpha]}\left(t_{1}^{[\alpha]}, \ldots, t_{k}^{[\alpha]}\right)$.

Since $f^{[\alpha]}$ is a partial function, $t^{[\alpha]}$ is not always defined. 
The set $\|t \sim q\|$ was defined in Definition 2.7 for a general algebraic model. Next lemma specifies it for the Beth model.

\section{Lemma 3.11}

Suppose $t$ is an evaluated term of sort $\pi$ and $q \in D_{\pi}$. Then

1) $\left\{\alpha \mid t^{[\alpha]}=q\right\}$ is an open set;

2) $\|t \sim q\|=C\left(\left\{\alpha \mid t^{[\alpha]}=q\right\}\right)$.

\section{Proof}

1) Proof is by induction on the complexity of $t$.

If $t$ is an element of $D_{\pi}$, then $\left\{\alpha \mid t^{[\alpha]}=q\right\}$ is either $T$ or $\perp$; in both cases it is an open set.

When $t$ is a constant $c$, the proof is similar.

Suppose $t$ is $f\left(t_{1}, \ldots, t_{k}\right)$, where each $t_{i}$ is an evaluated term of sort $\pi_{i}, i=1, \ldots, k$.

Then $f^{[\alpha]}\left(t_{1}^{[\alpha]}, \ldots, t_{k}^{[\alpha]}\right)=q$ implies that each $t_{i}^{[\alpha]}$ is defined.

So

$$
\begin{aligned}
& \left\{\alpha \mid t^{[\alpha]}=q\right\}=\bigcup_{\substack{q_{1} \in D_{\pi_{1}}, \ldots, \ldots \\
q_{k} \in D_{\pi_{k}}}}\left(\left\{\alpha \mid f^{[\alpha]}\left(q_{1}, \ldots, q_{k}\right)=q\right\}\right. \\
& \left.\bigcap\left\{\alpha \mid t_{1}^{[\alpha]}=q_{1}\right\} \cap \ldots \cap\left\{\alpha \mid t_{k}^{[\alpha]}=q_{k}\right\}\right) .
\end{aligned}
$$

By condition (4) of the definition of Beth model, $\{\alpha \mid$ $\left.f^{[\alpha]}\left(q_{1}, \ldots, q_{k}\right)=q\right\}$ is an open set. Each $\left\{\alpha \mid t_{i}^{[\alpha]}=q_{i}\right\}$ is an open set by the inductive assumption.

Therefore $\left\{\alpha \mid t^{[\alpha]}=q\right\}$ is an open set.

2) Proof is by induction on the complexity of $t$.

If $t$ is an element of $D_{\pi}$, then $t^{[\alpha]}=t$. So each side of the equality is $\begin{cases}T & \text { if } t=q, \\ \perp & \text { otherwise. }\end{cases}$

If $t$ is a constant $c$, then $t^{[\alpha]}=\bar{c}$. So each side of the equality is $\left\{\begin{array}{l}T \quad \text { if } \bar{c}=q, \\ \perp \text { otherwise. }\end{array}\right.$.

Suppose $t$ is $f\left(t_{1}, \ldots, t_{k}\right)$, where each $t_{i}$ is an evaluated term of sort $\pi_{i}, i=1, \ldots, k$.

Then

$$
\begin{gathered}
\left\{\alpha \mid t^{[\alpha]}=q\right\}=\bigcup_{\substack{q_{1} \in D_{\pi_{1}} \\
\ldots, \bar{q}_{k} \in D_{\pi_{k}}}}\left\{\alpha \mid t_{1}^{[\alpha]}=q_{1} \& \ldots \& t_{k}^{[\alpha]}=q_{k}\right. \\
\left.\& f^{[\alpha]}\left(q_{1}, \ldots, q_{k}\right)=q\right\} .
\end{gathered}
$$

By the inductive assumption each $\left\|t_{i} \sim q_{i}\right\|=$ $C\left(\left\{\alpha \mid t_{i}^{[\alpha]}=q_{i}\right\}\right)$. By part 1), each $\left\{\alpha \mid t_{i}^{[\alpha]}=q_{i}\right\}$ is an open set. Then by Lemma 3.5.3):

$$
\begin{aligned}
& \widehat{F n}\left(f, q_{1}, \ldots, q_{k}, q\right) \wedge\left\|t_{1} \sim q_{1}\right\| \wedge \ldots \wedge\left\|t_{k} \sim q_{k}\right\| \\
& =\boldsymbol{C}\left(\left\{\alpha \mid f^{[\alpha]}\left(q_{1}, \ldots, q_{k}\right)=q\right\}\right) \\
& \cap \boldsymbol{C}\left(\left\{\alpha \mid t_{1}^{[\alpha]}=q_{1}\right\}\right) \cap \ldots \cap \boldsymbol{C}\left(\left\{\alpha \mid t_{k}^{[\alpha]}=q_{k}\right\}\right) \\
& =\boldsymbol{C}\left(\left\{\alpha \mid f^{[\alpha]}\left(q_{1}, \ldots, q_{k}\right)=q\right\}\right. \\
& \left.\cap\left\{\alpha \mid t_{1}^{[\alpha]}=q_{1}\right\} \cap \ldots \cap\left\{\alpha \mid t_{k}^{[\alpha]}=q_{k}\right\}\right) \\
& =\boldsymbol{C}\left(\left\{\alpha \mid t_{1}^{[\alpha]}=q_{1} \& \ldots \& t_{k}^{[\alpha]}=q_{k}\right.\right. \\
& \left.\left.\& f^{[\alpha]}\left(q_{1}, \ldots, q_{k}\right)=q\right\}\right) .
\end{aligned}
$$

So

$$
\begin{aligned}
& \|t \sim q\|=\bigvee\left\{\widehat{F n}\left(f, q_{1}, \ldots, q_{k}, q\right) \wedge\left\|t_{1} \sim q_{1}\right\| \wedge \ldots\right. \\
& \left.\wedge\left\|t_{k} \sim q_{k}\right\| \mid q_{1} \in D_{\pi_{1}}, \ldots, q_{k} \in D_{\pi_{k}}\right\} \\
& =\vee\left\{\boldsymbol { C } \left(\left\{\alpha \mid t_{1}^{[\alpha]}=q_{1} \& \ldots \& t_{k}^{[\alpha]}=q_{k}\right.\right.\right. \\
& \left.\left.\left.\& f^{[\alpha]}\left(q_{1}, \ldots, q_{k}\right)=q\right\}\right) \mid q_{1} \in D_{\pi_{1}}, \ldots, q_{k} \in D_{\pi_{k}}\right\} \\
& =\boldsymbol{C} \bigcup_{\substack{q_{1} \in D_{\pi_{1}}, \ldots, q_{k} \in D_{\pi_{k}}}}\left\{\alpha \mid t_{1}^{[\alpha]}=q_{1} \& \ldots \& t_{k}^{[\alpha]}=q_{k}\right. \\
& \left.\left.\& f^{[\alpha]}\left(q_{1}, \ldots, q_{k}\right)=q\right\}\right)=\boldsymbol{C}\left(\left\{\alpha \mid t^{[\alpha]}=q\right\}\right)
\end{aligned}
$$

by Lemma 3.8.2) and (6). $\square$

\section{Lemma 3.12}

For an evaluated term $t$ of sort $\pi$ :

$$
C\left(\bigcup_{q \in D_{\pi}}\left\{\alpha \mid t^{[\alpha]}=q\right\}\right)=T .
$$

\section{Proof}

By Lemma 2.8, $\vee\left\{\|t \sim q\| \mid q \in D_{\pi}\right\}=T$. So by Lemma 3.11.2) and Lemma 3.8.2),

$$
\begin{aligned}
T=\vee\left\{\boldsymbol{C}\left(\left\{\alpha \mid t^{[\alpha]}=q\right\}\right) \mid q \in D_{\pi}\right\} \\
=\boldsymbol{C}\left(\bigcup_{q \in D_{\pi}}\left\{\alpha \mid t^{[\alpha]}=q\right\}\right) .
\end{aligned}
$$




\section{Lemma 3.13}

Suppose $P\left(t_{1}, \ldots, t_{k}\right)$ is an atomic formula, where each $t_{i}$ is an evaluated term of sort $\pi_{i}, i=1, \ldots, k$.

Then:

$$
\begin{aligned}
& \left\|P\left(t_{1}, \ldots, t_{k}\right)\right\|=C\left(\bigcup_{\substack{q_{1} \in D_{\pi_{1}} \\
\ldots, q_{k} \in D_{\pi_{k}}}} \overline{\operatorname{Pr}}\left(P, q_{1}, \ldots, q_{k}\right)\right. \\
& \left.\bigcap\left\{\alpha \mid t_{1}^{[\alpha]}=q_{1} \& \ldots \& t_{k}^{[\alpha]}=q_{k}\right\}\right) .
\end{aligned}
$$

\section{Proof}

By Lemma 3.11.2):

$$
\begin{aligned}
& \widehat{\operatorname{Pr}}\left(P, q_{1}, \ldots, q_{k}\right) \wedge\left\|t_{1} \sim q_{1}\right\| \wedge \ldots \wedge\left\|t_{k} \sim q_{k}\right\| \\
& =\boldsymbol{C}\left(\overline{\operatorname{Pr}}\left(P, q_{1}, \ldots, q_{k}\right)\right) \cap \boldsymbol{C}\left(\left\{a \mid t_{1}^{[\alpha]}=q_{1}\right\}\right) \cap \ldots . \\
& \cap \boldsymbol{C}\left(\left\{\alpha \mid t_{k}^{[a]}=q_{k}\right\}\right) \\
& =\boldsymbol{C}\left(\overline{\operatorname{Pr}}\left(P, q_{1}, \ldots, q_{k}\right) \cap\left\{\alpha \mid t_{1}^{[\alpha]}=q_{1} \& \ldots \& t_{k}^{[\alpha]}=q_{k}\right\}\right) \text {. } \\
& \text { So }\left\|P\left(t_{1}, \ldots, t_{k}\right)\right\|=\vee\left\{\widehat{\operatorname{Pr}}\left(P, q_{1}, \ldots, q_{k}\right) \wedge\left\|t_{1} \sim q_{1}\right\| \wedge \ldots\right. \\
& \left.\wedge\left\|t_{k} \sim q_{k}\right\| \mid q_{1} \in D_{\pi_{1}}, \ldots, q_{k} \in D_{\pi_{k}}\right\} \\
& =\bigvee\left\{\boldsymbol { C } \left(\overline { \operatorname { P r } } ( P , q _ { 1 } , \ldots , q _ { k } ) \cap \left\{\alpha \mid t_{1}^{[\alpha]}=q_{1} \& \ldots\right.\right.\right. \\
& \left.\left.\left.\& t_{k}^{[\alpha]}=q_{k}\right\}\right) \mid q_{1} \in D_{\pi_{1}}, \ldots, q_{k} \in D_{\pi_{k}}\right\} \\
& =\boldsymbol{C}\left(\bigcup_{\substack{q_{1} \in D_{\pi_{1}} \\
\ldots, q_{k} \in D_{\pi_{k}}}}\left(\overline{\operatorname{Pr}}\left(P, q_{1}, \ldots, q_{k}\right) \cap\left\{\alpha \mid t_{1}^{[\alpha]}=q_{1} \& \ldots \& t_{k}^{[\alpha]}=q_{k}\right\}\right)\right)
\end{aligned}
$$

by Lemma 3.8.2).

\section{Definition 3.14}

A forcing relation $\Vdash$ is defined by:

for any $\alpha$ and evaluated formula $\varphi$

$$
\alpha \Vdash \varphi \Leftrightarrow \alpha \in\|\varphi\|
$$

\section{Theorem 3.15. Properties of the Forcing Relation.}

1) Monotonicity of forcing: $\alpha \Vdash \varphi \& \beta \leq \alpha \Rightarrow \beta \Vdash \varphi$.

2) $\alpha \Vdash \varphi \Leftrightarrow(\forall S \in Q(\alpha))(\exists \beta \in S)(\beta \Vdash \varphi)$.

3) Suppose $\varphi$ is an atomic formula $P\left(t_{1}, \ldots, t_{k}\right)$, where each $t_{i}$ is an evaluated term of sort $\pi_{i}, i=1, \ldots, k$. Then

$$
\begin{aligned}
& \alpha \Vdash \varphi \Leftrightarrow(\forall S \in \boldsymbol{Q}(\alpha))(\exists \beta \in S)\left(\exists q_{1} \in D_{\pi_{1}}\right) \ldots \\
& \left(\exists q_{k} \in D_{\pi_{k}}\right)\left[t_{1}^{[\beta]}=q_{1} \& \ldots \& t_{k}^{[\beta]}=q_{k} \& \beta \in \overline{\operatorname{Pr}}\left(P, q_{1}, \ldots, q_{k}\right)\right] .
\end{aligned}
$$

4) $\alpha \Vdash \varphi \wedge \psi \Leftrightarrow(\alpha \Vdash \varphi) \&(\alpha \Vdash \psi)$

5) $\alpha \Vdash \varphi \vee \psi \Leftrightarrow(\forall S \in Q(\alpha))(\exists \beta \in S)[(\beta \Vdash \varphi)$ or $(\beta \Vdash \psi)]$

6) $\alpha \Vdash \varphi \supset \psi \Leftrightarrow(\forall \beta \leq \alpha)(\beta \Vdash \varphi \Rightarrow \beta \Vdash \psi)$

7) $\alpha \Vdash \perp \Leftrightarrow \perp$

8) $\alpha \Vdash \neg \varphi \Leftrightarrow(\forall \beta \leq \alpha) \neg(\beta \Vdash \varphi)$

9) $\alpha \Vdash \forall x \varphi(x) \Leftrightarrow(\forall \beta \leq \alpha)\left(\forall q \in D_{\pi}\right)(\beta \Vdash \varphi(q))$, where $x$ is a variable of sort $\pi$

10) $\alpha \Vdash \exists x \varphi(x) \Leftrightarrow(\forall S \in Q(\alpha))(\exists \beta \in S)\left(\exists q \in D_{\pi}\right)(\beta$ $\Vdash \varphi(q))$, where $x$ is a variable of sort $\pi$

\section{Proof}

In the Beth model $\|\varphi\| \in C l(O p(M))$, so $\|\varphi\|$ is both an open set and a complete element.

1) Follows from the fact that $\|\varphi\|$ is an open set

2) Follows from the fact that $\|\varphi\|$ is a complete element

3) Follows from Lemma 3.13

The rest follow from Definition 2.9 and Theorem 3.7.

\section{Axiomatic Theory of Functionals of High Types}

As an application of the topological definition of Beth model, we construct a Beth model for an intuitionistic theory $S L P$ of functionals of high types. We introduced this theory in (Kachapova, 2015) and constructed its Beth model using the van Dalen's relational definition (Van Dalen, 1978). The topological definition outlined here simplifies some parts of the construction and proofs.

We define the theory $S L P$ in three steps: We introduce axiomatic theories $L, L P$ and $S L P$. In these theories variables have superscripts for types. A superscript for a variable is usually omitted when the variable is used for the second time or more in a formula (so its type is clear).

\subsection{Axiomatic Theory $L$}

The language of theory $L$ has the following variables: $x, y, z, \ldots$ over natural numbers (variables of type 0 ) and variables of type $n(n \geq 1)$ :

$F^{n}, G^{n}, H^{n}, \ldots$ over $n$-functionals (functionals of type $n$ );

$A^{n}, B^{n}, C^{n}, \ldots$ over lawlike $n$-functionals;

$\mathscr{F}^{n}, \mathcal{G}^{n}, \mathscr{H}^{n}, \ldots$ over lawless $n$-functionals. 
Constants: 0 of type 0 and for each $n \geq 1$ a constant $K^{n}$ (an analog of 0 for type $n$ ).

Functional symbols: $N^{n}, A p^{n}(n \geq 1), S$ for successor, and + .

Predicate symbols: $={ }_{n}$ for each $n \geq 0$.

Terms and $n$-functionals are defined recursively as follows.

1. Every numerical variable is a term

2. Constant 0 is a term

3. Every variable of type $n$ is an $n$-functional

4. Constant $K^{n}$ is an $n$-functional

5. If $t$ is a term, then $S t$ is a term

6. If $t_{1}$ and $t_{2}$ are terms, then $t_{1}+t_{2}$ and $t_{1} \cdot t_{2}$ are terms

7. If $Z$ is an $n$-functional, then $N^{n}(Z)$ is an $n$-functional (a successor of $Z$ )

8. If $Z$ is a 1 -functional, $t$ is a term, then $A p^{1}(Z, t)$ is a term

9. If $Z$ is an $(n+1)$-functional, $t$ is a term, then $A p^{n+1}(Z$, $t$ ) is an $n$-functional

$A p^{n}(Z, t)$ is interpreted as the result of application of functional $Z$ to term $t$. We also denote $A p^{n}(Z, t)$ as $Z(t)$.

Here 1-functional is interpreted as a function from natural numbers to natural numbers and $(n+1)$ functional is interpreted as a function from natural numbers to $n$-functionals.

Atomic formulas:

$t={ }_{0} \tau$, where $t$ and $\tau$ are terms;

$Z={ }_{n} V$, where $Z$ and $V$ are $n$-functionals $(n \geq 1)$.

Formulas are constructed from atomic formulas using logical connectives and quantifiers. For a formula $\varphi$ its sort $\operatorname{sort}(\varphi)$ is the maximal type of parameters in $\varphi$; it is 0 if $\varphi$ has no parameters.

The theory $L$ has intuitionistic predicate logic $H P C$ with equality axioms and the following non-logical axioms.

1. $S x \neq 0, S x=S y \supset x=y$.

2. $x+0=x, x+S y=S(x+y)$.

3. $x \cdot 0=0, x \cdot S y=x \cdot y+x$.

4. Induction for natural numbers:

$\varphi(0) \wedge \forall x(\varphi(x) \supset \varphi(S x)) \supset \forall x \varphi(x)$,

where $\varphi$ is any formula of $L$.

The axioms 1 - 4 define arithmetic at the bottom level.

5. $K^{n+1}(x)=K^{n}, \neg\left(N^{n}\left(F^{n}\right)=K^{n}\right)$

6. $N^{n+1}\left(F^{n+1}\right)(x)=N^{n}(F(x)), N^{n}\left(F^{n}\right)=N^{n}\left(G^{n}\right) \supset F^{n}=G^{n}$

The axioms 5 and 6 describe $K^{n}$ and $N^{n}$ as analogs of zero and successor function, respectively, on level $n$.
7. Principle of primitive recursive completeness of lawlike functions:

$$
\exists A^{1} \forall x(A(x)=t),
$$

where $t$ is any term containing only variables of type 0 and variables over lawlike 1-functionals.

Denote $L_{s}$ the fragment of the theory $L$ with types not greater than $s$. The language of $L_{1}$ has one type of functionals and is essentially the language of the intuitionistic analysis FIM.

\subsection{Axiomatic Theory $L P$}

This theory is obtained from $L$ by adding predicate symbols and axioms for the "creating subject".

Gödel numbering of symbols and expressions can be defined for the language of $L$. For an expression $q$ we denote $\llcorner q\lrcorner$ the Gödel number of $q$ in this numbering.

The language of theory $L P$ is the language of $L$ with an extra predicate symbol $P v_{\llcorner\bar{X}, \varphi\lrcorner}(z, \bar{X})$ for every formula $\varphi$ of $L$, which has all its parameters in the list $\bar{X}$; here $\bar{X}$ is a list of variables $X_{1}, \ldots, X_{k}$. Traditionally this symbol is denoted $\vdash_{z} \varphi(\bar{X})$; it means that the formula $\varphi(\bar{X})$ has been proven by the "creating subject" at time $z$.

Axioms of the theory $L P$ are all axioms of $L$, where the axiom schemata are taken for the formulas of the new language, and the following three axioms; in all of them $\varphi$ is an arbitrary formula of $L$.

Axioms for the "creating subject":

$$
\begin{aligned}
& \left(\vdash_{z} \varphi\right) \vee \neg\left(\vdash_{z} \varphi\right) ; \\
& \left(\vdash_{z} \varphi\right) \supset\left(\vdash_{z+y} \varphi\right) ; \\
& \exists z\left(\vdash_{z} \varphi\right) \equiv \varphi .
\end{aligned}
$$

The language of $L P_{s}$ is the language of $L_{s}$ with extra predicate symbols $P v_{\llcorner\bar{X} . \varphi\lrcorner}(z, \bar{X})$, where both $\varphi$ and $\bar{X}$ belong to the language of $L_{s}$. Axioms of the theory $L P_{s}$ are all axioms of $L P$, which are formulas of the language $L P_{s}$.

If $(A)$ is a formula of $L P$, we denote $\left(A_{S}\right)$ the version of $(A)$ with types not greater than $s$.

\subsection{Axiomatic Theory $S L P$}

We can introduce finite sequences in $L P$ and for any $k$-functional $F$ use the notation $\bar{F}(n)=<F(0), \ldots$, $F(n-1)>$. We consider the following three axioms for lawless functionals. 
$(L L 1)$ The axiom of existence of lawless functionals:

$$
\forall x \forall F^{n} \exists \mathscr{F}^{n}(\forall y \leq x)(\mathscr{F}(y)=F(y)) .
$$

(LL2) $\mathfrak{F}^{\mathfrak{F}^{n}}=\mathcal{G}^{n} \vee \mathcal{F}^{n} \neq \mathcal{G}^{n}$.

(LL3) The principle of open data:

$$
\varphi\left(\mathscr{K}^{n}\right) \supset \exists x \forall \mathcal{G}^{n}[\overline{\mathcal{G}}(x)=\overline{\mathscr{H}}(x) \supset \varphi(\mathcal{G})],
$$

where $\varphi$ is a formula of $L P, \operatorname{sort}(\varphi) \leq n$ and $\varphi$ does not have non-lawlike parameters of type $n$ other than $\mathscr{K}^{n}$.

Denote $(L L)$ a conjunction of the closures of $(L L 1)$, (LL2) and (LL3).

For an $m$-functional $F$ of language $L P$ and $n \leq m$ we denote $F(\underbrace{(0) \ldots(0)}_{n}$ as $F(0)^{n}$ for brevity. We consider the following two choice axioms.

$(C 1)$ The axiom of choice for numbers:

$$
\forall x \exists y \varphi(x, y) \supset \exists F^{m} \forall x \varphi\left(x, F(x)(0)^{m-1}\right),
$$

where $\varphi$ is a formula of $L P$ and $m \geq \max (\operatorname{sort}(\varphi), 1)$.

$(C 2)$ The axiom of choice with uniqueness:

$$
\forall x \exists ! G^{n}(x, G) \supset \exists F^{m} \forall x \varphi\left(x, F(x)(0)^{m-n-1}\right),
$$

where $\varphi$ is a formula of $L P$ and $m \geq \max (\operatorname{sort}(\varphi), n+1)$. (C2).

Denote $(C)$ a conjunction of the closures of $(C 1)$ and

\section{Bar induction axiom:}

$$
\begin{aligned}
& \text { (BI) } \quad \forall F^{\mathrm{l}} \exists x \varphi(\bar{F}(x)) \wedge \forall x \forall y[\varphi(y) \supset \varphi(y *\langle x\rangle)] \\
& \wedge \forall y[\forall x \psi(y *\langle x\rangle) \supset \psi(x)] \wedge \forall y[\varphi(y) \supset \psi(y)] \supset \psi(0) .
\end{aligned}
$$

Axiomatic theory $S L P$ is defined by:

$$
S L P=L P+(L L)+(C)+(B I)
$$

In the name of the theory $S L P, S$ stands for strong, $L$ for lawless and $P$ for proof (relating to the "creating subject").

The fragment $S L P S$ is defined by:

$$
S L P_{s}=L P_{s}+\left(L L_{s}\right)+\left(C_{s}\right)+\left(B I_{s}\right) .
$$

\section{Application of the Topological Beth Model}

As an application, we construct a Beth model for any fragment $S L P_{s}(s \geq 1)$ of the theory $S L P$. This is sufficient for the proof of consistency of $S L P$, since any formal proof in the theory $S L P$ is finite and therefore it is a proof in some fragment $S L P_{s}$.

\subsection{Notations}

A sequence of elements $x_{0}, x_{1}, \ldots, x_{n}$ is denoted $x=\left\langle x_{0}, x_{1}, \ldots, x_{n}\right\rangle$ and we denote $\operatorname{lth}(x)=n+1$.

The symbol $*$ denotes the concatenation function:

$$
\begin{aligned}
& \left\langle x_{0}, x_{1}, \ldots, x_{n}\right\rangle *\left\langle y_{0}, y_{1}, \ldots, y_{k}\right\rangle \\
& =\left\langle x_{0}, x_{1}, \ldots, x_{n}, y_{0}, y_{1}, \ldots, y_{k}\right\rangle .
\end{aligned}
$$

For a sequence $x,\langle x\rangle_{n}$ denotes its $n^{\text {th }}$ element and $\bar{x}(n)$ denotes the initial segment of $x$ of length $n$ for any $n<\operatorname{lth}(x)$. So if $x=\left\langle x_{0}, x_{1}, \ldots, x_{m-1}\right\rangle$ and $n \leq m$, then $\bar{x}(n)$ $=\left\langle x_{0}, x_{1}, \ldots, x_{n-1}\right\rangle$.

For a function $f$ on natural numbers, $\bar{f}(n)$ denotes the initial segment of $f$ of length $n$, that is the sequence $\langle f(0)$, $f(1), \ldots, f(n-1)\rangle$.

For a function $f$ of two variables denote

$$
f^{(x)}=\lambda y \cdot f(x, y) .
$$

Next we introduce a few notations for a fixed set $b$.

1. $b^{(n)}$ is the set of all sequences of elements of $b$ that have length $n$.

2. $b^{*}$ is the set of all finite sequences of elements of $b$.

3. On the set $b^{*}$ a partial order $\leq$ is defined by the following:

$$
y \leq x \text { if } x \text { is an initial segment of } y .
$$

With this order $b^{*}$ is a tree growing down; its root is the empty sequence $<>$.

Suppose $\langle d, \leq\rangle$ is a partially ordered set and $f:(d \times \omega) \rightleftharpoons c$.

1. $f$ is called monotonic on $d$ if for any $x, y \in d$, $\left(y \leq x \Rightarrow f^{(x)} \subseteq f^{(y)}\right)$.

2. $f$ is called complete on $d$ if for any path $S$ in $d$, $\mathrm{U}\left\{f^{(x)} \mid x \in S\right\}$ is a total function on $\omega$.

\subsection{Beth Model $\mathscr{B}_{s}$ for the Language $L_{s}$}

Fix $s \geq 1$. To construct a Beth model $\mathcal{B}_{s}$ for the language $L_{s}$, we will specify a tree $\langle M, \preceq\rangle$, a domain function $D$ and functions $\widehat{C n s t}, \overline{F n}$ and $\overline{P r}$.

1) First we introduce a triple of objects $\left\langle a_{k}, d_{k}, \preceq_{k}\right\rangle$ by induction on $k$; here $\preceq_{k}$ is a partial order on $d_{k}$. 
- $a_{0}=\omega$, the set of all natural numbers

- $d_{0}=\left\{\langle x\rangle \mid x \in a_{0}^{*}\right\}$; thus, each element of $d_{0}$ is a sequence of length 1

- $\preceq_{0}$ is generated by the order $\leq$ on $a_{0}^{*}$

$\langle x\rangle \preceq_{0}\langle y\rangle$ if $x \leq y$, that is the sequence $y$ is an initial segment of the sequence $x$.

For $\langle x\rangle \in d_{0}$ we denote $\operatorname{lh}(\langle x\rangle)=\operatorname{lth}(x)$ and call it the length of $\langle x\rangle$.

For $k \geq 1$ :

- $\quad a_{k}=\left\{f \mid f:\left(d_{k-1} \times \omega\right) \rightleftharpoons a_{k-1}\right.$ and $f$ is complete and monotonic $\}$;

- $d_{k}=\bigcup_{m=0}^{\infty}\left(a_{0}^{(m)} \times a_{1}^{(m)} \times \ldots \times a_{k}^{(m)}\right)$;

- $\preceq_{k}$ is defined by:

$x \preceq_{k} y$ if for each $i \leq k,\langle x\rangle_{i} \leq\langle y\rangle_{i}$, that is the sequence

$\langle y\rangle_{i}$ is an initial segment of the sequence $\langle x\rangle_{i}$.

If $x \in a_{0}^{(m)} \times \ldots \times a_{k}^{(m)}$, we denote $\operatorname{lh}(x)=m$ and call it the length of $x$.

We take $M=d_{s-1}$ with partial order $\preceq_{s-1}$, which we denote just $\preceq$.

With this order $M$ is a subtree of the direct product of trees $a_{0}{ }^{*}, \ldots, a_{s-1}{ }^{*} . M$ is a non-countable tree growing down. Its root is $\varepsilon=<\underbrace{<>, \ldots,<>>}_{s}$.

1) Next we define a domain for each sort of variables.

a) Domain for natural numbers is $a_{0}=\omega$.

b) Domain for $k$-functionals is $a_{k}(k=1,2, \ldots, s)$.

c) Domain for lawlike $k$-functionals is

$$
b_{k}=\left\{f \in a_{k} \mid f^{(<>)} \text {is a total function }\right\} .
$$

d) Before defining a domain for lawless functionals we introduce an auxiliary set $c_{k}(k \geq$ 1):

$c_{k}=\left\{\xi \mid \xi:\left(\omega \times a_{k-1}\right) \rightarrow a_{k-1}\right.$ and $\forall n\left(\xi^{(n)}\right.$ is a bijection on $\left.\left.a_{k}\right)\right\}$.

Elements of $c_{k}$ are called $k$-permutations. For any $k$ permutation $\xi$ we define $v_{k}(\xi)$ as the function $f \in a_{k}$ such that for any $n \in \omega, x \in d_{k-1}$ :

$$
f(x, n)=\left\{\begin{array}{cc}
\xi^{(n)}\left(\left\langle\langle x\rangle_{k-1}\right\rangle_{n}\right) & \text { if } n<\operatorname{lh}(x), \\
\text { undefined } & \text { otherwise. }
\end{array}\right.
$$

Domain for lawless $k$-functionals is:

$$
l_{k}=\left\{v_{k}(\xi) \mid \xi \in c_{k}\right\}, k=1,2, \ldots, s .
$$

Clearly, $b_{k} \subseteq a_{k}$ and $l_{k} \subseteq a_{k}, k=1, \ldots, s$. For any $\alpha$ and $k<s, \operatorname{lh}(\alpha)=\operatorname{lth}\left(\langle\alpha\rangle_{k}\right)$.

2) Next we define $\widehat{C n s t}$, that is interpretation $\bar{C}$ for each constant $C$.

a) $\overline{0}=0$.

b) The interpretation of constant $K^{k}(k=1, \ldots, s)$ is given by the following:

$$
\begin{gathered}
\bar{K}^{1}(x, n)=0 \text { for any } n \in \omega \text { and } x \in d_{0} ; \\
\bar{K}^{k+1}(x, n)=\bar{K}^{k} \text { for any } n \in \omega \text { and } x \in d_{k} .
\end{gathered}
$$

3) Next we define $\overline{F n}$. This means defining a partial function $h^{[\alpha]}$ for each functional symbol $h$ and $\alpha \in M$.

a) If $\theta$ is $\cdot$ or + , then $\theta^{[\alpha]}=\theta$.

b) A successor function $S^{k}$ of type $k(k=0,1, \ldots, s)$ is defined by:

$$
S^{0}(x)=x+1 ; \quad S^{n+1}(f)=S^{n} \circ f .
$$

Functional symbol $S$ is interpreted by: $S^{[\alpha]}=S^{0}$. Functional symbol $N^{k}$ is interpreted by: $N^{k[\alpha]}=S^{k}$.

c) Functional symbol $A p^{k}$ is interpreted by:

$A p^{k[\alpha]}(f, n)=f(\bar{\alpha}(k), n)$ for any $n \in \omega, f \in a_{k}, k=1, \ldots, s$.

Thus, at each node $\alpha$ the values of a $k$-functional $f$ depend not on the entire $\alpha$ but only on its first $k$ components.

Due to the definition of $a_{k}$, the conditions (4) and (5) of the Definition 3.9 of Beth model are satisfied.

4) The only predicate symbols in the language $L$ are equalities $=_{k}(k=0,1,2 \ldots)$.

$\overline{P r}$ is defined by:

$$
\overline{\operatorname{Pr}}\left(p={ }_{k} q\right)=\left\{\begin{array}{cc}
T & \text { if } p=q, \\
\perp & \text { otherwise }
\end{array}\right.
$$

For any $p, q \in a_{k}$. Clearly, $\overline{\operatorname{Pr}}\left(p={ }_{k} q\right)$ is an open set.

This completes the definition of the model $\mathcal{B}_{S}$ for the language of $L_{s}$. 
For the general case of Beth model we already defined:

- $Z^{[\alpha]}$, where $Z$ an evaluated term or $n$-functional (Definition 3.10) and

- $\quad$ the forcing relation $\alpha \Vdash \varphi$ (Definition 3.14)

\subsection{Extending the Model $\mathscr{B}_{\mathrm{s}}$ to the Language $S L P_{\mathrm{s}}$}

First we extend the model to the language $L P_{s}$. For that we need to interpret the extra predicate symbols $P v_{\llcorner\bar{X} . \varphi\lrcorner}(z, \bar{X})$, where both $\varphi$ and $X$ belong to the language of $L_{s}$.

\section{Definition 5.1}

Consider a formula $\varphi$ of $L_{s}$ with all its parameters in the list $X_{1}, \ldots, X_{k}$, which is denoted $\bar{X}$ in short. For brevity we denote the predicate $P v_{\llcorner\bar{X} . \varphi\lrcorner}(z, \bar{X})$ as $P_{0}(z, \bar{X})$. We define:

$$
\overline{\operatorname{Pr}}\left(P_{0}, n, \bar{q}\right)=\{\alpha \mid \exists \gamma[\alpha \preceq \gamma \& \operatorname{lh}(\gamma)=n \& \gamma \Vdash \varphi(\bar{q})]\},
$$

where, $\bar{q}$ is the list $q_{1}, \ldots, q_{k}$.

Let us show that $\overline{\operatorname{Pr}}\left(P_{0}, n, \bar{q}\right)$ is an open set in the order topology. Suppose $\alpha \in \overline{\operatorname{Pr}}\left(P_{0}, n, \bar{q}\right)$. Then there is $\gamma$ such that $\alpha \preceq \gamma \& \operatorname{lh}(\gamma)=n \& \gamma \Vdash \varphi(\bar{q})$. Then for any $\beta \preceq \alpha$ we have $\beta \preceq \gamma$, so $\beta \in \overline{\operatorname{Pr}}\left(P_{0}, n, \bar{q}\right)$.

The language of $S L P_{s}$ is the same as the language $L P_{s}$, so the extended model $\mathcal{B}_{S}$ is also a Beth model for the language of $S L P$.

\section{Lemma 5.2}

$\alpha \Vdash P_{0}(n, \bar{q}) \Leftrightarrow(\forall S \in \boldsymbol{Q}(\alpha))(\exists \gamma \in S)[\operatorname{lh}(\gamma)=n \& \gamma \Vdash \varphi(\bar{q})]$.

\section{Proof}

Proof follows from the definitions. $\square$

\subsection{Soundness of the Model $\mathscr{B}_{s}$ for the Theory $S L P_{s}$}

Theorem 5.3. Soundness Theorem.

$$
S L P_{s} \vdash \varphi \Rightarrow \mathscr{B}_{s} \Vdash \bar{\varphi} .
$$

\section{Proof}

Proof is by induction on the length of derivation of $\varphi$. Here we provide proofs only for the case of the axioms $(C S 1)-(C S 3)$ for the "creating subject". We prove these in all detail to illustrate our application of the topological Beth model. Other, more technical proofs can be found in (Kachapova, 2014; 2015).
We use the notations of Definition 5.1.

$$
\text { Proof of } \mathscr{B}_{S} \Vdash\left(\overline{C S 1_{s}}\right) \text {. }
$$

$(C S 1)$ is $\left(\vdash_{z} \varphi\right) \vee \neg\left(\vdash_{z} \varphi\right)$. It is sufficient to prove:

$$
\varepsilon \Vdash P_{0}(n, \bar{q}) \vee \neg P_{0}(n, \bar{q}) .
$$

By Theorem 3.15.5) it is equivalent to:

$$
(\forall S \in \boldsymbol{Q}(\varepsilon))(\exists \alpha \in S)\left[\alpha \Vdash P_{0}(n, \bar{q}) \text { or } \alpha \Vdash \neg P_{0}(n, \bar{q})\right] \text {. }
$$

Consider a path $S \in Q(\varepsilon)$. Fix $\alpha \in S$ with $\operatorname{lh}(\alpha)=n$. There are two cases: $\alpha \Vdash \varphi(q)$ or $\alpha \not \neq \varphi(q)$ (we use classical logic in metamathematics).

Case 1. $\alpha \Vdash \varphi(\bar{q})$.

Since $\operatorname{lh}(\alpha)=n$, by Lemma 5.2 we have $\alpha \Vdash P_{0}(n, \bar{q})$.

Case 2. $\alpha \not \varphi(\bar{q})$.

We will show that in this case $\alpha \Vdash \neg P_{0}(n, \bar{q})$. By Theorem 3.15.8), it is equivalent to:

$$
(\forall \beta \preceq \alpha) \neg\left(\beta \Vdash P_{0}(n, \bar{q})\right) .
$$

Suppose $\beta \Vdash P_{0}(n, \bar{q})$ for some $\beta \preceq \alpha$. Consider an arbitrary $S_{1} \in Q(\beta)$. By Lemma 5.2, there is $\gamma \in S_{1}$ such that $l h(\gamma)=n \& \gamma \Vdash \varphi(\bar{q})$.

Since $S_{1} \in \boldsymbol{Q}(\beta) \& \beta \preceq \alpha$, we have $\alpha \in S_{1}$. Both $\alpha, \gamma \in S_{1}$ and $\operatorname{lh}(\alpha)=n=\operatorname{lh}(\gamma)$, so $\alpha=\gamma$ and $\alpha \Vdash \varphi(\bar{q})$.

Contradiction. Therefore $\neg\left(\beta \Vdash P_{0}(n, \bar{q})\right)$ :

$$
\text { Proof of } \mathcal{B}_{s} \Vdash\left(\overline{C S 2_{s}}\right) \text {. }
$$

$(C S 2)$ is $\left(\vdash_{z} \varphi\right) \supset\left(\vdash_{z+y} \varphi\right)$. It is sufficient to prove:

$$
\varepsilon \Vdash P_{0}(n, \bar{q}) \supset P_{0}(n+m, \bar{q}) .
$$

Fix $\alpha$. Suppose $\alpha \Vdash P_{0}(n, \bar{q})$. Consider $S \in Q(\alpha)$. By Lemma 5.2, there is $\gamma \in S$ such that $l h(\gamma)=n$ and $\gamma \Vdash \varphi(\bar{q})$.

There is $\beta \in S$ with $l h(\beta)=n+m$. Since $\beta \preccurlyeq \gamma$, we have $\beta \Vdash \varphi(\bar{q})$ by monotonicity of forcing (Theorem 3.15.1)). This proves $\alpha \Vdash P_{0}(n+m, \bar{q})$. 


$$
\text { Proof of } \mathscr{B}_{s} \Vdash\left(\overline{C S 3_{s}}\right) \text {. }
$$

$(C S 3)$ is $\exists z\left(\vdash_{z} \varphi\right) \equiv \varphi$.

It is sufficient to prove: $\varepsilon \Vdash \exists z P_{0}(z, \bar{q}) \equiv \varphi(\bar{q})$, which is equivalent to

$$
\varepsilon \Vdash \exists z P_{0}(z, \bar{q}) \supset \varphi(\bar{q})
$$

and

$$
\varepsilon \Vdash \varphi(\bar{q}) \supset \exists z P_{0}(z, \bar{q}) .
$$

\section{Proof of (7)}

Suppose for some $\alpha$,

$$
\alpha \Vdash \exists z P_{0}(z, \bar{q}) .
$$

We need to prove $\alpha \Vdash \varphi(\bar{q})$. Consider a path $S \in \boldsymbol{Q}(\alpha)$. By (9), there is $\beta \in S$ and $n$ such that $\beta \Vdash P_{0}(n, \bar{q})$. Then $S \in \boldsymbol{Q}(\beta)$ and by Lemma 5.2, there is $\gamma \in S$ with $\gamma \Vdash \varphi(\bar{q})$. Thus,

$$
(\forall S \in \boldsymbol{Q}(\alpha))(\exists \gamma \in S)(\gamma \Vdash \varphi(\bar{q})) .
$$

By Theorem 3.15.2), $\alpha \Vdash \varphi(\bar{q})$.

\section{Proof of (8)}

Suppose for some $\alpha, \alpha \Vdash \varphi(\bar{q})$. Take $n=\operatorname{lh}(\alpha)$. Suppose $S \in \boldsymbol{Q}(\alpha)$. For any $S_{1} \in \boldsymbol{Q}(\alpha)$ there is $\gamma=\alpha$ with $\operatorname{lh}(\gamma)=n \& \gamma \Vdash \varphi(\bar{q})$; so by Lemma 5.2, $\alpha \Vdash P_{0}(n, \bar{q})$. Hence $\alpha \Vdash \exists z P_{0}(z, \bar{q})$.

\section{Conclusion}

In this study we describe detailed steps of construction of Beth model from the topological point of view. The model is applied to intuitionistic functionals of high types and the theory SLP that includes lawless functionals, "the creating subject", bar induction and some choice axioms; the model is used to prove the consistency of SLP. The topological version of Beth model and our model, in particular, can potentially be useful to investigate other intuitionistic principles with respect to functionals of high types.

\section{Acknowledgement}

The author thanks the Editor in Chief and anonymous Reviewer.

\section{Ethics}

This is a mathematical article; no ethical issues can arise after its publication.

\section{References}

Dragalin, A. G. (1987). Mathematical Intuitionism. Introduction to Proof Theory. American Mathematical Society, USA.

Kachapova, F. (2014). A strong intuitionistic theory of functionals. arXiv:1403.2813.

Kachapova, F. (2015). A strong multi-typed intuitionistic theory of functionals. The Journal of Symbolic Logic, 80(3), 1035-1065.

Moschovakis, J. R. (1973). A topological interpretation of second-order intuitionistic arithmetic. Compositio Mathematica, 26(3), 261-275.

Moschovakis, J. R. (1987). Relative lawlessness in intuitionistic analysis. The Journal of Symbolic Logic, 52(1), 68-88.

van Dalen, D. (1978). An interpretation of intuitionistic analysis. Annals of Mathematical Logic, 13(1), 1-43. 